\title{
THE EFFECT OF COLLATERAL LIGAMENT INJURY ON CARTILAGE CONTACT IN KNEE JOINTS MODELED WITH SIX DEGREES OF FREEDOM
}

\author{
NERIMAN ÖZADA \\ Department of Mechanical Engineering \\ Eastern Mediterranean University \\ Gazimagusa, North Cyprus, Turkey \\ neriman.ozada@emu.edu.tr
}

Received 21 April 2015

Accepted 5 October 2015

Published 28 March 2016

\begin{abstract}
The purpose of this study was to create a kinematic model of the knee joint with six degrees of freedom (DOF) and evaluate the effect of medial collateral ligament (MCL) and lateral collateral ligament (LCL) rupture on cartilage contact point distribution on the tibia during flexion. We hypothesized that collateral ligament contributions vary over six DOF of knee joint articulation and affect the cartilage contact point distribution during joint articulation. The ligament contributions and distribution of joint cartilage contact points cannot be fully assessed with simplified joint models or invasive experiments. Therefore, we developed a new model in which the tibia and femur centers of mass were determined from their surface geometry, and the displacement of the moving tibia was determined from the displacements of the attached ligaments. Compared to the intact knee, the tibia with the LCL removed had higher medial translation and lower valgus rotation. The tibia with the MCL removed had higher lateral translation and higher valgus rotation than the intact knee. At $0^{\circ}, 30^{\circ}$, and $60^{\circ}$, the tibia with the LCL removed had more internal rotation than the intact knee. Understanding six DOF knee joint kinematics with integration of ligament contributions and cartilage contact positions is useful for the diagnosis of ligament injuries and the design of articulating surfaces for total arthroplasty.
\end{abstract}

Keywords: Knee; joint; cartilage contact; kinematics.

\section{Introduction}

A vast number of biomechanical studies have been performed to better understand the complete kinematics and contact mechanics of anatomical and artificial knee joints. This has contributed to improvements in our understanding of normal and pathological joint kinematics and contact mechanics and has helped in the development of replacement implants that mimic native knee joint kinematics.

This is an Open Access article published by World Scientific Publishing Company. It is distributed under the terms of the Creative Commons Attribution 4.0 (CC-BY) License. Further distribution of this work is permitted, provided the original work is properly cited. 
Experimental and clinical tools including dynamic stereo X-ray, ${ }^{1}$ magnetic resonance imaging (MRI), and fluoroscopic imaging ${ }^{2}$ and software such as the OpenSim musculoskeletal model, LifeModeler, Software for Interactive Musculoskeletal Modeling (SIMM) are available for the evaluation of tibiofemoral joint kinematics and measuring articular contact locations.

However, most methods do not provide kinematic information in all six degrees of freedom (DOF) so that it can be correlated with cartilage contact positions during joint articulation. Nevertheless, commercial and non-commercial biomechanical analysis software can be used to predict joint kinematics and contact mechanics that are difficult to observe experimentally due to their invasive nature. ${ }^{3,4}$ The representation of joint kinematics differs in translation and rotation according to the DOF, with a joint with six DOF and no limit to kinematic motion regarded as unconstrained. However, most biomechanical joint modeling studies restrict joint motion by defining permanent or dependent constraints. Kinematically constrained joints are known as "idealized" or "bilateral" joints, in which the contact geometry between articulating bodies is not included in motion analysis. Thus, unilateral joint models are used to introduce contact in the analysis using state-dependent kinematic constraints. Bilateral and unilateral joint models rarely possess six DOF during articulation, and due to the simplified modeling approaches used in multi-body dynamics-based software, precise joint models have yet to be developed. For example, LifeModeler ${ }^{5}$ is an ADAMS software plugin that is used for multi-body dynamic analysis of human body joints, but even this software uses a unilateral-type contact with reduced kinematic DOF when the contact is active in total knee replacement (TKR) analysis.

Therefore, joint modeling tools and software still fail to fully represent unconstrained anatomical joints and consider the contact surface geometry. This hampers comprehensive analysis of the kinematics and contact mechanics of injured, deteriorated, and artificial joints. Although software based on finite element analysis may model joints in six DOF, it is impractical for clinical use due to the computational cost. Furthermore, software based on multi-body dynamics are limited to constrained joints and no programs are currently capable of predicting unconstrained kinematics and their relationship with contact surface geometry.

A number of studies have evaluated six DOF kinematics of knee joints with anterior cruciate ligament (ACL) and medial collateral ligament (MCL) deficiencies. However, studies on altered six DOF kinematics of knees with MCL and lateral collateral ligament (LCL) deficiencies are limited. ${ }^{6}$ Several dual-orthogonal fluoroscopy and MRI-based biomechanical studies have resulted in comprehensive joint models and have quantified contact locations based on bone and cartilage surface geometry, ${ }^{2,7}$ and Wang et al. ${ }^{8}$ recently studied contact locations during the stance phase of simulated walking. However, none of these studies have revealed how collateral ligament rupture affects joint cartilage contact positions.

The purpose of the present study was to model the knee joint with six DOF to study MCL and LCL function. We used multi-body dynamics-based joint modeling software $^{9}$ and calculated the tibial and femoral centers of mass based on surface 
mesh data obtained by bone surface scanning. We find that calculating the center of mass of intact and artificial bones distinguishes the effect of geometry in joint kinematics and cartilage contact positions. Six DOF kinematics and the cartilage contact distribution were calculated for an intact knee and knees without the MCL or LCL, and relative changes between the MCL and LCL lengths and joint cartilage contact positions on the tibia were evaluated. In doing so, we present the complete kinematics and cartilage contact point distribution on the tibia during six DOF joint articulation, overcoming the limitations of current experiments or simplified joint modeling approaches.

\section{Materials and Methods}

\subsection{Knee joint model construction}

A left knee joint model was developed that included the tibia and femur but not the patella and fibula. Bone geometries were obtained using a laser scanner and manipulated with software (Geomagic, Morrisville, NC, USA ${ }^{10}$ ). The tibia and femur were modeled as rigid bodies (tibia mobile and femur stationary).

The main knee joint ligaments (MCL, LCL, and anterior and posterior cruciate ligaments) and articular cartilage were included in the model. Muscles and tendons were excluded and passive joint flexion was modeled. The ligaments were modeled as three-dimensional linear elastic spring elements with only tension forces included. The spring stiffness $(k)$ of the ligaments (MCL, 65 N/mm; LCL, $60 \mathrm{~N} / \mathrm{mm}$ ) was determined based on published data ${ }^{5}$ and was assumed to be axisymmetric. Only one articular cartilage model was placed between the tibia and femur, which was modeled with three-dimensional linear viscoelastic spring-dashpot elements $(k, 40 \mathrm{~N} / \mathrm{mm}$; damping value $[c], 400 \mathrm{Ns} / \mathrm{mm})$.

A Cartesian coordinate system was used to establish local and global coordinate systems (translations along the $x-, y$-, and $z$-axes; rotations around axes: $\alpha$ [x-axis], $\beta$ [y-axis], and $\gamma[z$-axis]; Fig. 1). Cardinal reference planes also were used (translation: $+x$, anterior; $-x$, posterior; $+y$, lateral; $-y$, medial; $+z$, superior; $-z$, inferior and rotation: $+\alpha$, varus [adduction]; $-\alpha$, valgus [abduction]; $+\beta$, flexion; $-\beta$, extension; $+\gamma$, internal rotation; $-\gamma$, external rotation).

\subsection{Joint modeling theory}

The inertial properties, center of mass, center of rotation, coordinate systems, and kinematic properties were software defined to derive dynamic equations of motion. The center of mass was calculated for each bone from the surface geometry using an algorithm combined with the Gauss Divergence Theorem as developed by Mirtich ${ }^{11}$ The center of mass of the tibia was defined as $\mathrm{o}_{t}$, and the center of mass of the femur was defined as $\mathrm{O}_{\mathrm{f}}$, which coincided with the local reference axes (Fig. 1). The displacement of the moving tibia was derived from the displacements of the attached ligaments, and the centers of mass of the tibia and femur were determined from the surface geometry. Therefore, six DOF joint kinematics were derived without 


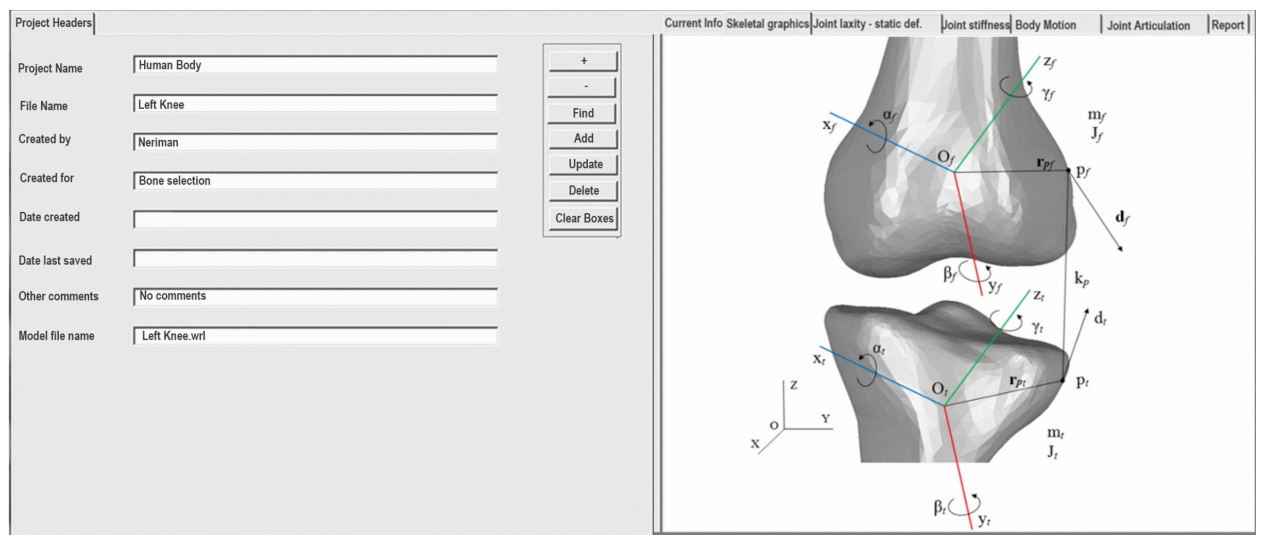

Fig. 1. Knee joint model and coordinate systems.

reducing the DOF during joint articulation. The displacement of each ligament was described from the translations, rotations, and positions of the attachment points. The displacement equations of the ligaments, modeled as springs attached to tibia and femur, were calculated from:

$$
\begin{gathered}
\mathbf{d}_{t}=\mathbf{x}_{t}+\boldsymbol{\theta}_{t} \times \mathbf{r}_{p t}, \\
\mathbf{d}_{f}=\mathbf{x}_{f}+\boldsymbol{\theta}_{f} \times \mathbf{r}_{p f},
\end{gathered}
$$

where $p t$ and $p f$ were the spring attachment points on the tibia and femur, $\mathbf{d}_{t}$ and $\mathbf{d}_{f}$ were the local displacements of the spring attachment points, $\mathbf{r}_{p t}$ and $\mathbf{r}_{p f}$ were the position vectors of these attachment points, and $\mathbf{x}_{t}, \mathbf{x}_{f}, \boldsymbol{\theta}_{t}$, and $\boldsymbol{\theta}_{f}$ were the translations and rotations of an attached spring. The six DOF joint kinematics were determined by describing the body displacements from the displacement of each ligament.

The mechanical and kinematic quantities, displacement and moment equations, and coordinate systems were used to construct Newton-Euler linear dynamic equations of motion. All displacement and moment equations were obtained from the center of mass of the tibia and femur. Transformation matrices were used to determine the relative motion of the tibia and femur, and the assembled linear dynamic equations of motion of the tibia and femur in a global coordinate system were calculated using:

$$
\begin{gathered}
{\left[\begin{array}{cc}
\mathbf{m}_{t} & 0 \\
0 & \mathbf{J}_{t}
\end{array}\right]\left\{\begin{array}{c}
\ddot{\mathbf{x}}_{t} \\
\ddot{\boldsymbol{\theta}}_{t}
\end{array}\right\}-\left[\begin{array}{cc}
\mathbf{k}_{p} & \mathbf{k}_{p} \mathbf{R}_{p t} \\
\mathbf{R}_{p t}^{\mathrm{T}} \mathbf{k}_{p} & \mathbf{R}_{p t}^{\mathrm{T}} \mathbf{k}_{p} \mathbf{R}_{p t}
\end{array}\right]\left\{\begin{array}{l}
\mathbf{x}_{t} \\
\boldsymbol{\theta}_{t}
\end{array}\right\}+\left[\begin{array}{cc}
\mathbf{k}_{p} & \mathbf{k}_{p} \mathbf{R}_{p f} \\
\mathbf{R}_{p t}^{\mathrm{T}} \mathbf{k}_{p} & \mathbf{R}_{p t}^{\mathrm{T}} \mathbf{k}_{p} \mathbf{R}_{p f}
\end{array}\right]\left\{\begin{array}{l}
\mathbf{x}_{t} \\
\boldsymbol{\theta}_{f}
\end{array}\right\}=\left\{\begin{array}{l}
\mathbf{F}_{t} \\
\mathbf{M}_{t}
\end{array}\right\}} \\
{\left[\begin{array}{cc}
\mathbf{m}_{f} & 0 \\
0 & \mathbf{J}_{f}
\end{array}\right]\left\{\begin{array}{c}
\ddot{\mathbf{x}}_{f} \\
\ddot{\boldsymbol{\theta}}_{f}
\end{array}\right\}+\left[\begin{array}{cc}
\mathbf{k}_{p} & \mathbf{k}_{p} \mathbf{R}_{p t} \\
\mathbf{R}_{p t}^{\mathrm{T}} \mathbf{k}_{p} & \mathbf{R}_{p t}^{\mathrm{T}} \mathbf{k}_{p} \mathbf{R}_{p t}
\end{array}\right]\left\{\begin{array}{c}
\mathbf{x}_{t} \\
\boldsymbol{\theta}_{t}
\end{array}\right\}-\left[\begin{array}{cc}
\mathbf{k}_{p} & \mathbf{k}_{p} \mathbf{R}_{p f} \\
\mathbf{R}_{p f}^{\mathrm{T}} \mathbf{k}_{p} & \mathbf{R}_{p f}^{\mathrm{T}} \mathbf{k}_{p} \mathbf{R}_{p f}
\end{array}\right]\left\{\begin{array}{c}
\mathbf{x}_{f} \\
\boldsymbol{\theta}_{f}
\end{array}\right\}} \\
=\left\{\begin{array}{l}
\mathbf{F}_{f} \\
\mathbf{M}_{f}
\end{array}\right\},
\end{gathered}
$$


where $\mathbf{m}$ is mass, $\mathbf{J}$ is the moment of inertia, $\mathbf{R}$ is the matrix form of the position vector $\mathbf{r}, \mathbf{k}_{p}$ is the spring stiffness, $\mathbf{x}$ is translation, $\boldsymbol{\theta}$ is rotation, $\mathbf{F}$ is the force vector acting along the line of action, and $\mathbf{M}$ is the moment vector. The transposed $\mathbf{R}$ matrix was denoted $\mathbf{R}^{\mathrm{T}}$.

\subsection{Minimum mass (point) algorithm}

Line of action and moment-arm estimates are important for accurate joint moment calculations. A minimum mass (point) algorithm based on heuristic and surface geometry was used to generate the tissue (ligament) line of action based on points distributed along the bone surfaces. Tissue attachment points were selected to generate the tissue line of action: the origin was selected as point $\mathrm{p}_{\mathrm{t}}$ (Fig. 2), and then a local cylindrical coordinate system was defined by the orthogonal vectors $\mathbf{u}$, $\mathbf{v}$, and $\mathbf{w}$, with the reference at the origin.

The insertion was selected as point $\mathbf{p}_{f}$, and a straight line $\mathbf{L}$ was drawn between the origin and insertion. Furthermore, the tibia and femur were sliced into equal segments along vector $\mathbf{L}$ with $10^{\circ}$ between each segment. The total number of surface points was calculated to select a segment suitable for drawing the tissue line of action, and the segment that had the least number of surface points was selected. When necessary, projection points were used to count the total number of points at each segment and a projection angle was used to determine where a point belonged. Point $\mathbf{p}$ was selected on the femur, and its projections $\mathbf{p}_{u}$ and $\mathbf{p}_{w}$ in the $\mathbf{u}$ and $\mathbf{w}$ planes and projection angle $\alpha_{t}$ were determined (Fig. 2). The projections and projection angles were determined using:

$$
\begin{gathered}
p_{u}=\left(\mathbf{p}_{t}-\mathbf{p}_{f}\right) \cdot \mathbf{u}, \\
p_{w}=\left(\mathbf{p}_{t}-\mathbf{p}_{f}\right) \cdot \mathbf{w}, \\
\alpha_{i}=\tan ^{-1} \frac{p_{w}}{p_{u}} .
\end{gathered}
$$

After choosing a segment for the tissue line of action, a convex hull algorithm was used to wrap the line around the bone surfaces between the attachment points (Fig. 2).

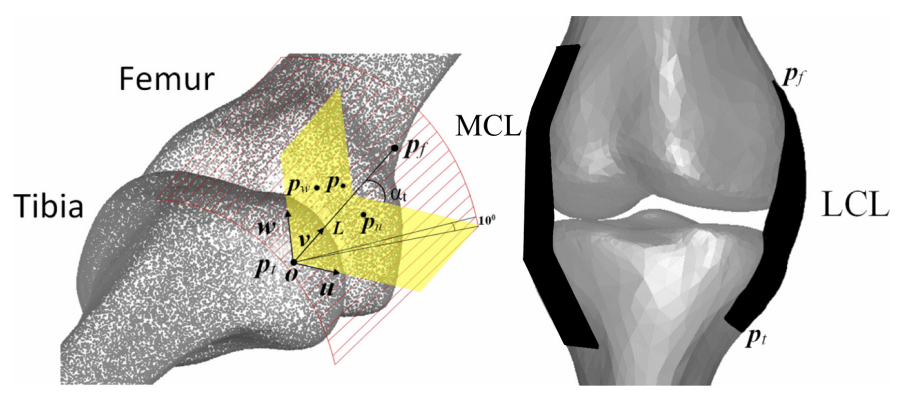

Fig. 2. Minimum mass (point) algorithm for the tibia and femur with wrapped LCL. 
All theories and algorithms were composed using the musculoskeletal joint modeler software. ${ }^{9}$ Bone files were imported into the program in the Virtual Reality Modeling Language file format. The initial conditions and parameters were calculated including mass, inertia, and the local coordinate systems of the tibia and femur from the centers of mass. The ligament attachment points $\left(\mathbf{p}_{t}\right.$ and $\left.\mathbf{p}_{f}\right)$ were selected. Tensile forces were applied along the ligament lines of action between $\mathbf{p}_{t}$ and $\mathbf{p}_{f}$, and the equilibrium condition of the knee was adjusted at each flexion angle $\left(0^{\circ}, 30^{\circ}\right.$, $60^{\circ}, 90^{\circ}, 120^{\circ}$, and $150^{\circ}$ ). This procedure was applied for each flexion angle to determine the displacement of each ligament and the tibia.

\subsection{Collision detection and response algorithms}

After establishing the MCL and LCL lines of action and equilibrium positions, collision detection and collision response algorithms were next performed. These algorithms are also based on the tibia and femur contact surface geometry. First, velocity $\mathbf{V}_{t}$ was applied to tibia to move it toward the femur. Then, potential collision points $\mathbf{p}_{t}$ and $\mathbf{p}_{f}$ on the tibia and femur, respectively, were detected based on the shortest distance constraint equation point coordinates. Points that were not potentially colliding were eliminated and excluded from the collision response algorithm.

After obtaining potentially colliding point pairs, a spring-dashpot element was attached along each shortest distance among $\mathbf{l}_{t f}$ to represent the deformable cartilage model. This algorithm differs from the unilateral contact equations, since the tibia kinematics still retain six DOF even if the contact is active. In this way, the tibia can perform three DOF translation and three DOF rotation during every step of the analysis. To perform dynamic contact analysis, the contact force equation of Fisk and Wayne was adopted, which is a function of cartilage stiffness $k_{c}(8000 \mathrm{~N} /$ $\mathrm{mm})$, damping value $c(400 \mathrm{~N}-\mathrm{s} / \mathrm{mm})$, allowable surface point penetration at maximum damping value $d(0.001 \mathrm{~mm})$, average penetration depth $g$ between each point pair, and the exponent value of $e$ (set at 2). When penetration occurs between the point pairs, the average penetration depth is calculated at every step at $0^{\circ}, 30^{\circ}$, $60^{\circ}, 90^{\circ}, 120^{\circ}$, and $150^{\circ}$ of flexion; then, based on the depth, the force at each spring dashpot element is obtained. In this way the collision detection and response algorithms apply realistic contact dynamics modeling and provide realistic simulation of the knee joint. The cartilage contact points were selected based on the centroids of the intersection of the point pairs of the tibia and femur.

\subsubsection{Conditions}

Two sets of calculations were made: (1) six DOF kinematics of the knee joint and medial and lateral cartilage contact positions, and (2) the relationship between the medial and lateral cartilage contact positions with MCL and LCL length changes. Three different joint conditions were used in both calculations: intact, MCL removed, and LCL removed. 


\section{Results}

\subsection{Knee joint translation and cartilage contact position}

Due to the loss of knee joint stability, greater posterior translations of the tibial center of mass were seen with the MCL or LCL removed than in the intact knee. Compared to an intact knee, the average differences in anterior-posterior (AP) translation were $3 \%$ for the knee with the MCL removed and $6 \%$ for the knee with the LCL removed. Posterior translation of lateral cartilage contact was reduced $64 \%$ with the MCL removed and $26 \%$ with the LCL removed, while posterior translation of medial cartilage contact was reduced $3 \%$ with the MCL removed and $0.2 \%$ with the LCL removed (Table 1 and Fig. 3).

Table 1. Translation of the tibial center of mass with medial and lateral cartilage contact positions.

\begin{tabular}{|c|c|c|c|c|c|c|c|c|c|}
\hline \multirow[b]{2}{*}{ Knee flexion $\left(^{\circ}\right)$} & \multicolumn{9}{|c|}{$\begin{array}{l}\text { Tibial center of mass translation versus medial and lateral } \\
\text { cartilage contact positions }(\mathrm{mm})\end{array}$} \\
\hline & \multicolumn{3}{|c|}{ Intact knee } & \multicolumn{3}{|c|}{$\begin{array}{l}\text { Lateral cartilage } \\
\text { compartment }\end{array}$} & \multicolumn{3}{|c|}{$\begin{array}{l}\text { Medial cartilage } \\
\text { compartment }\end{array}$} \\
\hline \multicolumn{10}{|l|}{ (A) Intact knee } \\
\hline Axis & $x$ & $y$ & $z$ & $x$ & $y$ & $z$ & $x$ & $y$ & $z$ \\
\hline 0 & 0 & 0 & 0 & -0.84 & 19.95 & 129.57 & -2.8 & -18.03 & 127.5 \\
\hline 30 & -81.72 & 3.71 & 21.35 & -13.9 & 16.72 & 127.29 & -13.07 & -22.38 & 128.38 \\
\hline 60 & -130.81 & 4.67 & 62.57 & -19.53 & 16.94 & 134.44 & -27.22 & -26.63 & 137.29 \\
\hline 90 & -159.52 & 10.07 & 143.54 & -32.84 & 19.9 & 147.8 & -31.43 & -22.29 & 147.08 \\
\hline 120 & -154.32 & 10.99 & 212.89 & -21.18 & 17.33 & 159.91 & -28.76 & -24.05 & 162.46 \\
\hline \multirow[t]{2}{*}{150} & -117.04 & 6.68 & 258.26 & -19.63 & 13.27 & 156.15 & -22.09 & -27.11 & 163.79 \\
\hline & $\mathrm{MC}$ & removes & knee & \multicolumn{3}{|c|}{$\begin{array}{l}\text { Lateral cartilage } \\
\text { compartment }\end{array}$} & \multicolumn{3}{|c|}{$\begin{array}{l}\text { Medial cartilage } \\
\text { compartment }\end{array}$} \\
\hline
\end{tabular}

(B) MCL removed knee

\begin{tabular}{lrlrrrrrrr} 
Axis & $x$ & $y$ & $z$ & $x$ & $y$ & $z$ & $x$ & $y$ & $z$ \\
0 & 0.12 & 4.8 & -5.58 & -14.3 & 17.66 & 133.48 & -3.98 & -19.57 & 129.55 \\
30 & -79.79 & 8.86 & 13.83 & -23.49 & 20.35 & 144.43 & -23.61 & -22.79 & 137.01 \\
60 & -136.17 & 9.27 & 66.98 & -23.05 & 16.72 & 148.62 & -22.29 & -28.42 & 142.27 \\
90 & -163.38 & 23.45 & 141.91 & -18.99 & 12.23 & 158.29 & -33.41 & -30.48 & 154.53 \\
120 & -157.06 & 18.96 & 205.99 & -23.06 & 18.3 & 164.9 & -28.6 & -22.94 & 169.31 \\
150 & -117.54 & 10.88 & 255.17 & -25.56 & 18.67 & 162.26 & -25.49 & -25.43 & 170.29 \\
\hline
\end{tabular}

\begin{tabular}{ccc}
\hline LCL removed knee & $\begin{array}{c}\text { Lateral cartilage } \\
\text { compartment }\end{array}$ & $\begin{array}{c}\text { Medial cartilage } \\
\text { compartment }\end{array}$ \\
\hline
\end{tabular}

\begin{tabular}{lccccccccc}
\hline \multicolumn{2}{l}{$(\mathrm{C})$ LCL removed knee } & \multicolumn{1}{c}{} & & & & & & \\
Axis & $x$ & $y$ & $z$ & $x$ & $y$ & $z$ & $x$ & $y$ & $z$ \\
0 & 0.48 & -4.36 & -4.89 & -6.94 & 16.98 & 132.53 & -1.3 & -25.36 & 129.93 \\
30 & -81.42 & -1.83 & 15.24 & -19.73 & 14.48 & 141.26 & -22.12 & -24.84 & 137.87 \\
60 & -136.68 & 4.1 & 62.52 & -17.87 & 10.92 & 143.06 & -27.65 & -29.38 & 142.83 \\
90 & -163.2 & -2.13 & 141.79 & -26.2 & 14.63 & 147.72 & -29.89 & -35.55 & 151.5 \\
120 & -158.08 & -13.49 & 195.83 & -29.94 & 15.67 & 159.81 & -29.42 & -33.85 & 159.88 \\
150 & -120.46 & -15.48 & 250.9 & -28.65 & 15.95 & 165.51 & -24.37 & -30.71 & 165.32 \\
\hline
\end{tabular}




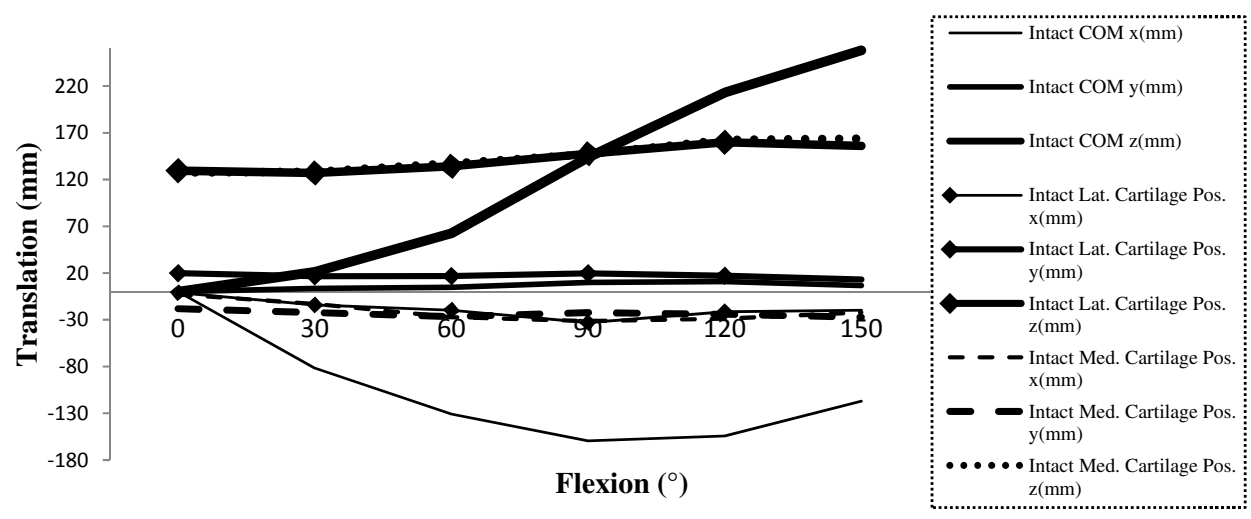

(a)

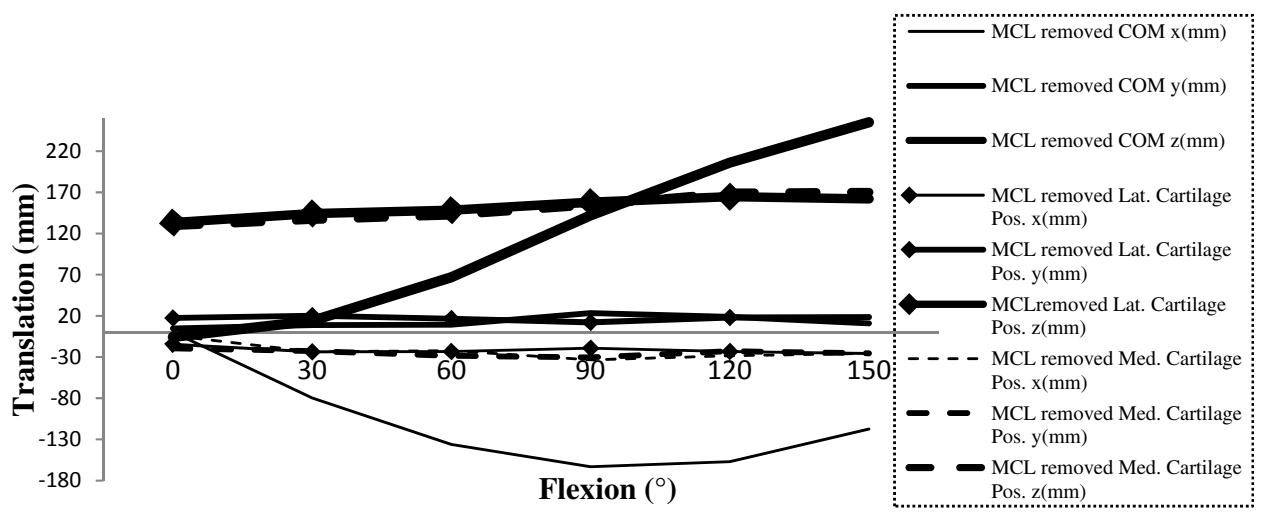

(b)

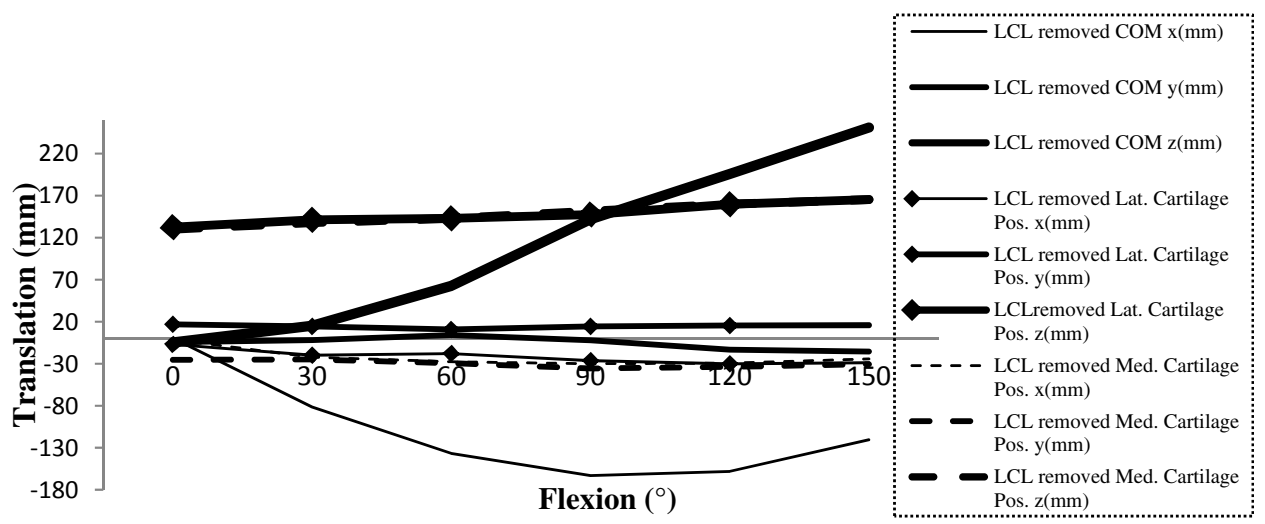

(c)

Fig. 3. Three DOF anterior-posterior (AP), medial-lateral (ML), superior-inferior (SI) translations versus cartilage contact positions for: (a) intact knee, (b) MCL-removed knee and (c) LCL-removed knee. 
The tibia with the MCL removed had the greatest lateral translation and the tibia with the LCL removed had the greatest medial translation. There was a large difference in ML translation of the tibia at $90^{\circ}$ flexion in intact, MCL-removed, and LCL-removed knees. Although the tibia in an intact knee had $10.1 \mathrm{~mm}$ lateral translation, the tibia with the MCL removed had $23.5 \mathrm{~mm}$ lateral translation and the tibia with the LCL removed had $-2.1 \mathrm{~mm}$ medial translation. Lateral translation of the lateral cartilage contact position was similar to the changes in the tibial center of mass. Compared to an intact knee, lateral translation of lateral cartilage contact increased by $21 \%$ with the MCL removed and decreased by $9 \%$ with the LCL removed. However, the medial cartilage compartment increased in medial translation: $17 \%$ with the LCL removed and $20 \%$ with the MCL removed.

With respect to SI translations, the average changes in SI translations of the tibia center of mass were $17 \%$ with the MCL removed and $14 \%$ with the LCL removed. Compared to an intact knee, superior translations of the lateral cartilage contact position increased $1 \%$ with the LCL removed and decreased $13 \%$ with the MCL removed. Superior translations of medial cartilage contact increased $12 \%$ with the MCL removed and decreased $2 \%$ with the LCL removed. Therefore, MCL removal markedly affected SI translation, while LCL removal markedly affected AP and ML translations.

\subsection{Knee joint rotation and cartilage contact position}

Removal of the collateral ligaments resulted in changes to varus-valgus (VV) rotation. MCL removal caused a $123 \%$ change in valgus rotation, and LCL removal caused $87 \%$ varus rotation between $0^{\circ}$ and $150^{\circ}$ flexion (Table 2 and Fig. 4).

Table 2. Rotations of the tibial center of mass with two DOF.

\begin{tabular}{|c|c|c|c|c|}
\hline \multirow[b]{2}{*}{ Knee flexion $\left(^{\circ}\right)$} & \multirow[b]{2}{*}{ Rotation axis } & \multicolumn{3}{|c|}{ Center of mass rotation $\alpha\left(^{\circ}\right)$} \\
\hline & & Intact knee & MCL removed & LCL removed \\
\hline \multicolumn{5}{|l|}{ (A) VV } \\
\hline 0 & $\alpha$ & 0 & -4 & 2 \\
\hline 30 & $\alpha$ & -2.63 & -5.35 & 1.06 \\
\hline 60 & $\alpha$ & -4.39 & -9.03 & -4.35 \\
\hline 90 & $\alpha$ & -7.15 & -14.04 & -7.01 \\
\hline 120 & $\alpha$ & -8.18 & -16.37 & -7.6 \\
\hline \multirow[t]{2}{*}{150} & $\alpha$ & -3.51 & -8.39 & -9.62 \\
\hline & & \multicolumn{3}{|c|}{ Center of mass rotation $\gamma\left({ }^{\circ}\right)$} \\
\hline Knee flexion $\left(^{\circ}\right)$ & Rotation axis & Intact knee & MCL removed & LCL removed \\
\hline \multicolumn{5}{|l|}{ (B) IE rotations } \\
\hline 0 & $\gamma$ & 0 & -0.4 & 0.5 \\
\hline 30 & $\gamma$ & 4.2 & 3.62 & 8.81 \\
\hline 60 & $\gamma$ & 10.85 & 13.89 & 16.39 \\
\hline 90 & $\gamma$ & 7.44 & 14 & 10.13 \\
\hline 120 & $\gamma$ & 13.97 & 17.14 & 7.4 \\
\hline 150 & $\gamma$ & 7.05 & 11.21 & 7.5 \\
\hline
\end{tabular}




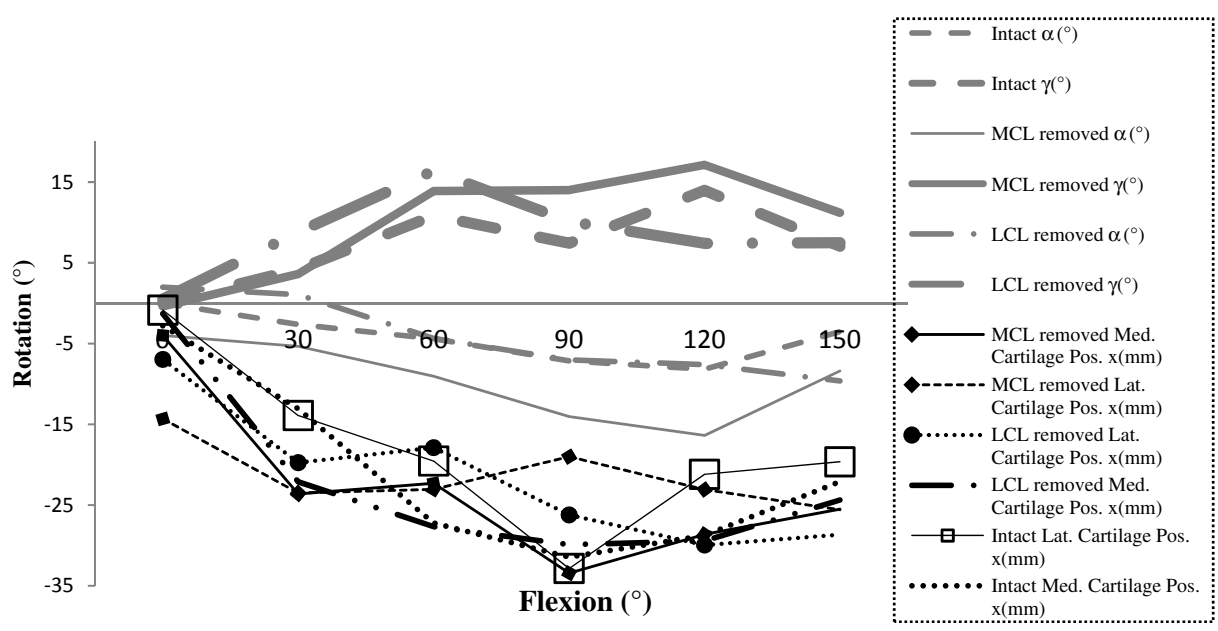

(a)
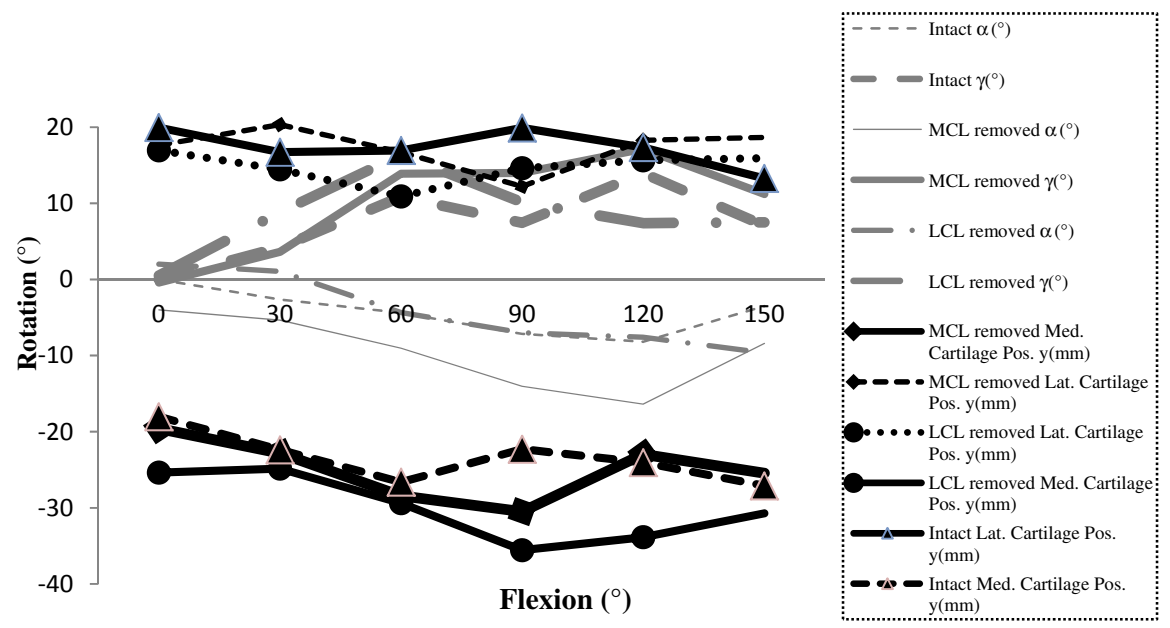

(b)

Fig. 4. VV and internal-external (IE) rotations of the tibial center of mass for the intact knee and knee with either MCL or LCL removed versus: (a) AP positions of cartilage contact and (b) ML positions of cartilage contact.

The increase in valgus rotation caused by MCL removal was also associated with an increase in lateral translation of the lateral cartilage contact and an increase in medial translation of the medial cartilage contact. Therefore, MCL removal resulted in wider lateral and medial cartilage contact positions. The increase in varus rotation associated with LCL removal had a similar but less influential effect on lateral and medial cartilage contact positions in the ML direction.

Changes in IE rotation were complex during knee flexion. Internal rotation was highest in the knee with the LCL removed between $0^{\circ}$ and $60^{\circ}$ flexion, resulting in 
similar medial cartilage contact position changes between $0^{\circ}$ and $60^{\circ}$ flexion in the intact knee and when the LCL was removed. However, a gradual decrease in internal rotation between $60^{\circ}$ and $150^{\circ}$ flexion resulted in medial compartment cartilage contact position movement from lateral to medial, opposite to the position change of the medial compartment seen in the intact knee. Compared to the intact knee, MCL removal resulted in a similar increase and decrease in internal rotation: an increase in internal rotation at $0^{\circ}, 30^{\circ}$, and $60^{\circ}$; a decrease at $90^{\circ}$; an increase at $120^{\circ}$; and a decrease at $150^{\circ}$. These changes mostly affected lateral translation of the lateral cartilage compartment, in which an increase in internal rotation at $0^{\circ}, 30^{\circ}$, and $60^{\circ}$ resulted in a decrease in lateral translation of the contact points. At $90^{\circ}$, lateral translation of the cartilage contact position increased in the intact knee but decreased with the MCL removed. LCL removal affected IE rotation of the knee to a greater degree than MCL removal: there were marked changes in IE rotation at $90^{\circ}$, which also resulted in marked changes in lateral cartilage posterior translation with the MCL removed and medial cartilage medial translation with the LCL removed.

\subsection{Collateral ligament length associated with cartilage contact position}

In the intact knee, the LCL length decreased when the knee was flexed between $0^{\circ}$ and $60^{\circ}$ and increased between $60^{\circ}$ and $120^{\circ}$ (Table 3 and Fig. 5). There was a

Table 3. Length of collateral ligaments in the intact knee and knees with the opposite ligament removed during flexion.

\begin{tabular}{lcc}
\hline & \multicolumn{2}{c}{ Length of LCL $(\mathrm{mm})$} \\
\cline { 2 - 3 } Knee flexion $\left(^{\circ}\right)$ & Intact knee & MCL removed \\
\hline (A) Length of LCL & & \\
0 & 36.02 & 41.86 \\
30 & 35.41 & 36.74 \\
60 & 31.44 & 33.51 \\
90 & 31.78 & 34.6 \\
120 & 35.58 & 32.66 \\
150 & 31.54 & 27.5 \\
\hline & Length of MCL $(\mathrm{mm})$ \\
\cline { 2 - 3 } Knee flexion $\left(^{\circ}\right)$ & Intact knee & LCL removed \\
\hline (B) Length of MCL & & \\
0 & 71.3 & 62.7 \\
30 & 71.47 & 63.1 \\
60 & 73.78 & 65.53 \\
90 & 66.21 & 72.39 \\
120 & 79.92 & 65.4 \\
150 & 68.43 & 58.71 \\
\hline
\end{tabular}




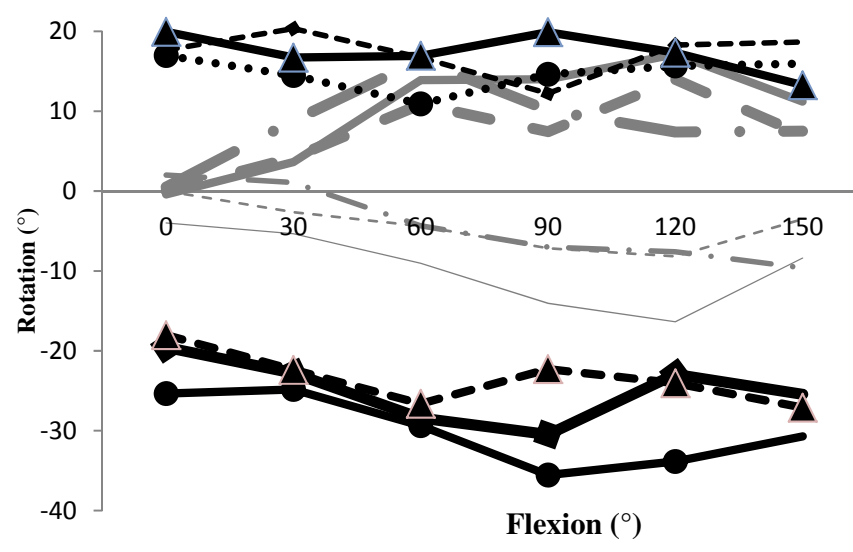

(a)

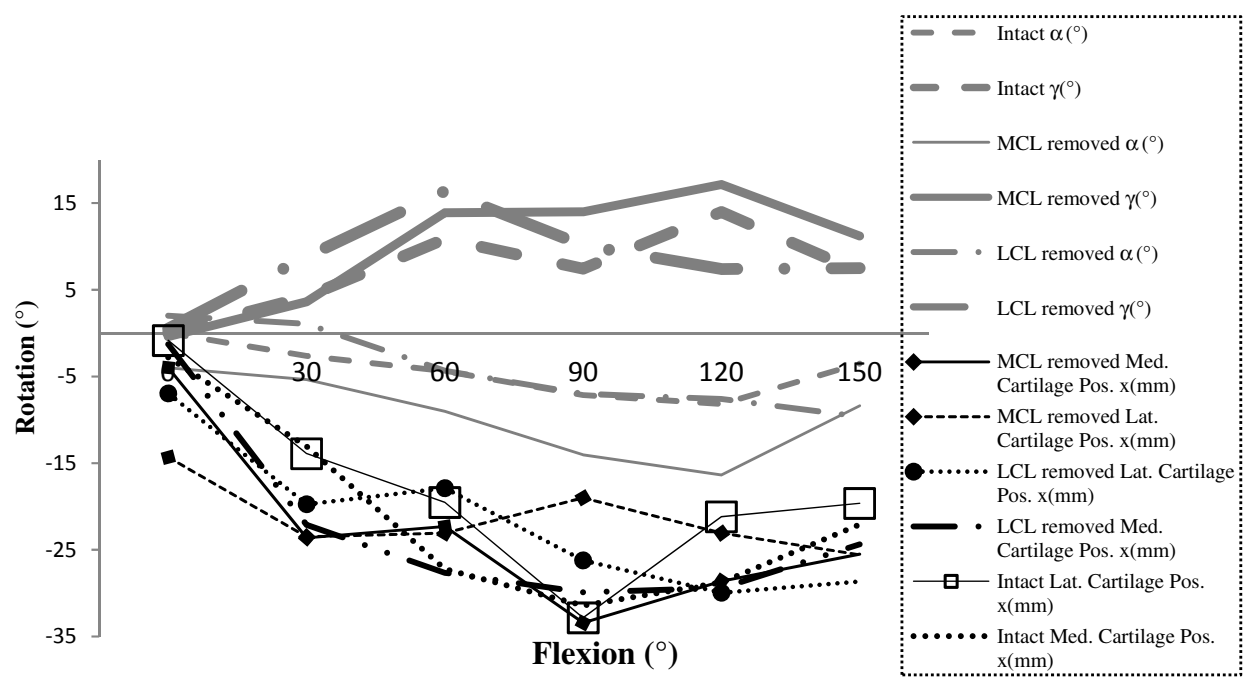

(b)

Fig. 5. Collateral ligament lengths versus: (a) lateral-medial translations of cartilage contact and (b) AP translations of cartilage contact for the intact knee and knee with either MCL or LCL removed.

similar variation in lateral translation of the lateral cartilage contact position. Compared to the intact knee, MCL removal resulted in an increase in the length of the LCL between $60^{\circ}$ and $90^{\circ}$ and a decrease between $90^{\circ}$ and $120^{\circ}$. This changed the lateral translations of both the lateral and medial cartilage compartments to medial translations at $90^{\circ}$ flexion. The maximum LCL length change was an $11 \%$ decrease between $120^{\circ}$ and $150^{\circ}$ in the intact knee, while the average change in LCL length was $9 \%$ between the intact knee and the knee with the MCL removed. The 
average increase in medial translations of the lateral and medial compartments was $37 \%$ with the MCL removed at $90^{\circ}$.

In the intact knee, the MCL length increased when the knee was flexed between $0^{\circ}$ and $60^{\circ}$, decreased between $60^{\circ}$ and $90^{\circ}$, increased between $90^{\circ}$ and $120^{\circ}$, and decreased between $120^{\circ}$ and $150^{\circ}$ (Table 3 and Fig. 5). In the knee with the LCL removed, the MCL length increased when the knee was flexed between $0^{\circ}$ and $90^{\circ}$ and decreased between $90^{\circ}$ and $150^{\circ}$. Compared with intact knee medial and lateral compartment cartilage contact positions, LCL removal increased the medial translations of the cartilage contact points in both compartments in the lateral to medial direction and increased posterior translation of the lateral cartilage contact point between $0^{\circ}$ and $60^{\circ}$ and increased anterior translation of the lateral cartilage contact between $60^{\circ}$ and $90^{\circ}$.

The maximum percentage change was a $20 \%$ increase in MCL length between $90^{\circ}$ and $120^{\circ}$ in the intact knee, which also showed the maximum anterior translation of the lateral cartilage position. The average difference in MCL length was $10 \%$ between the intact knee and the knee with the LCL removed. The MCL length was affected by LCL removal more than LCL length was affected by MCL removal.

\section{Discussion}

Here we present a six DOF kinematic analysis of the intact knee and knees with the MCL or LCL removed to investigate their contributions to knee joint stability. Knee joint translations and rotations varied on ligament removal. To better understand the behavior of the knee joint in totality, variations in contact positions of both the lateral and medial cartilage compartments were investigated and depicted during joint articulation. Combining six DOF kinematic analysis with analysis of the contribution of collateral ligaments and variations in cartilage contact positions reveal hitherto unknown information about the knee joint.

The intact knee and the knee with the MCL or LCL removed showed similar translational movements at some flexion angles (Fig. 3). However, MCL and LCL removal mostly affected AP and lateral-medial translations. In particular, collateral ligament removal affected posterior translation of the lateral cartilage compartment. SI translations between $60^{\circ}$ and $90^{\circ}$ and $\mathrm{AP}$ translations between $0^{\circ}$ and $60^{\circ}$ were identical for all knee conditions, suggesting that assessment of translational movements at particular degrees of flexion may not provide sufficient information to diagnose knee joint disorders. MCL and LCL removal mainly affected lateral-medial translations of the tibia, and, similarly, MCL removal was the main contributor to medial and lateral translations of cartilage contact points. To facilitate the diagnosis of MCL and LCL rupture, contact positions can also be utilized for specific translations and rotations in addition to kinematics knowledge.

To enable comparison of knee joint kinematics and collateral ligament length changes to previous work, the midpoints between the tibial epicondyles and femoral condyles were chosen as reference points for the tibia and femur, respectively. 


\section{N. Özada}

To enable comparison of translations of the lateral and medial cartilage compartments with previous work, the midpoints of each lateral and medial cartilage compartment were chosen. Our findings were consistent with tibial translational movements detected in previous studies (Fig. 6), ${ }^{12-15}$ with the tibia moving posteriorly and superiorly during full flexion. When the lateral and medial cartilage compartment translations were compared with translation of the tibia, the observed

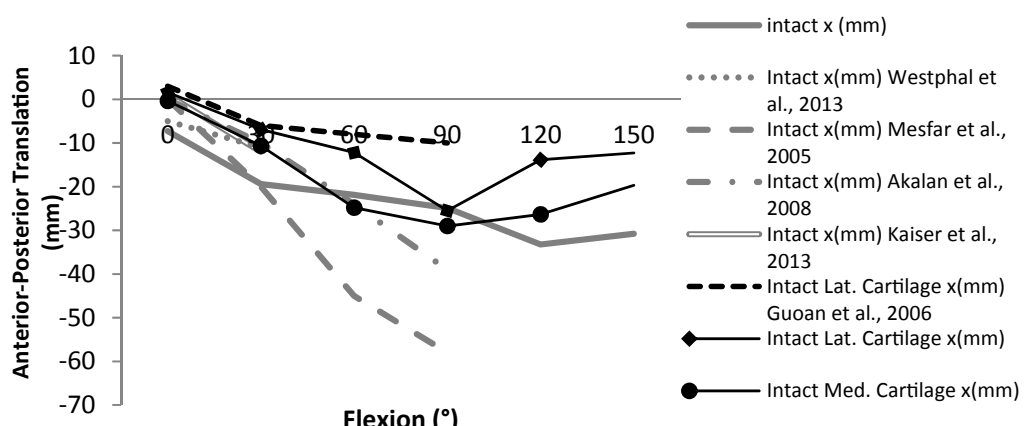

(a)

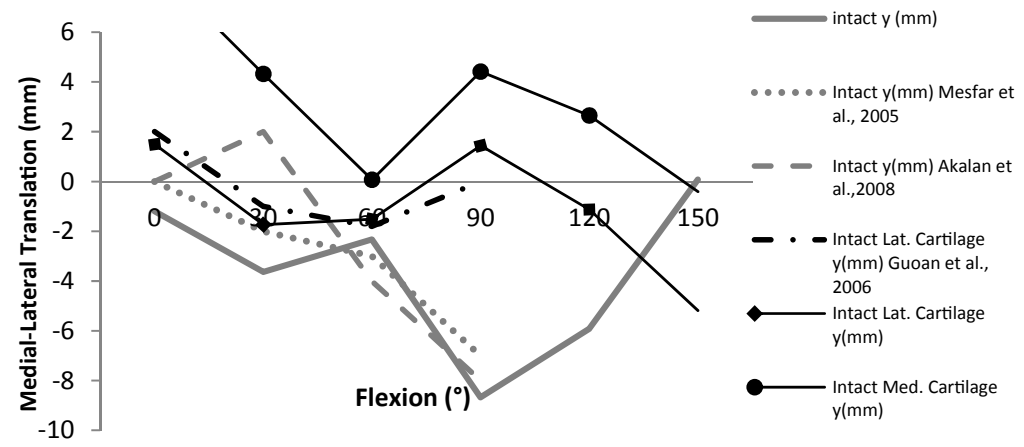

(b)

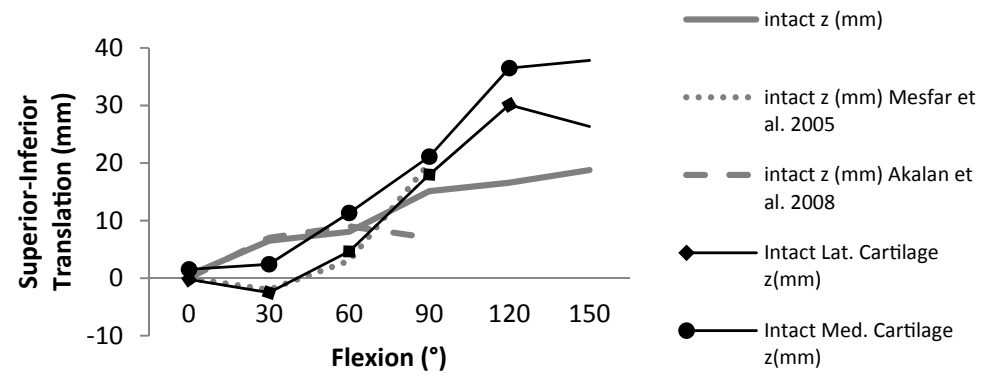

(c)

Fig. 6. Comparison of tibial translations with previous studies. ${ }^{12-16}$ (a) AP translation, (b) ML translation and (c) SI translation. 
posterior and superior translations of the lateral and medial cartilage compartments were consistent with the posterior and superior translations of the tibia during flexion. However, at $90^{\circ}$ flexion, posterior translation of the lateral compartment increased sharply; the shortest distance was probably detected near the midpoint of the tibial epicondyle at this point, highlighting the limitation of plotting only one contact point translation. Between $0^{\circ}$ and $90^{\circ}$ flexion, posterior translations of the lateral cartilage contact points were similar to previously published results ${ }^{16}$ The medial and lateral translations of the tibia and cartilage contact points changed in a similar manner based on the degree of flexion. However, between $0^{\circ}$ and $90^{\circ}$, the lateral and medial translations of the lateral cartilage compartment were similar to previous studies. $^{16}$

According to two DOF analysis for tibial rotation, a 3\% difference in VV rotation was observed between knees with intact and removed LCL between $60^{\circ}$ and $120^{\circ}$ flexion. Therefore, the LCL may be important in limiting VV rotation between $60^{\circ}$ and $120^{\circ}$ of flexion but may not affect rotation trajectory.

In the present study, the IE and VV rotations were similar in both intact knees and those with removed MCLs, although the magnitudes of IE and VV rotations of the tibia when the MCL was removed were much higher than tibial rotations in an intact knee. Therefore, MCL rupture may alter the amount but not the trajectory of $\mathrm{VV}$ and IE rotation. However the tibia with the LCL removed had the highest IE rotation between $0^{\circ}$ and $60^{\circ}$ and the lowest IE rotation between $90^{\circ}$ and $150^{\circ}$, suggesting that LCL rupture may increase tibial internal rotation between $0^{\circ}$ and $60^{\circ}$ and increase tibial external rotation between $90^{\circ}$ and $150^{\circ}$. We therefore compared our results on VV and IE rotations of the tibia in an intact knee with published data and plotted changes in lateral and medial cartilage contact positions with respect to tibial rotations. ${ }^{6,14,17-19}$ As shown in Fig. 7 , between $0^{\circ}$ and $90^{\circ}$ flexion, the trajectory of ML translation of the lateral cartilage contact position is similar to the trajectory of VV rotations in Refs. 6 and 14. Cartilage contact position correlated with VV rotation may therefore be a useful indicator of ligament injury.

LCL length and cartilage contact position changes in intact and MCL-removed knees were also measured. The LCL length increased from $0^{\circ}$ to $30^{\circ}$ in the intact knee but decreased in the knee with the MCL removed in an inversely proportional relationship (Fig. 5). Furthermore, LCL length in knees with intact and removed MCLs changed proportionally between $30^{\circ}$ and $90^{\circ}$ of flexion. However, between $90^{\circ}$ and $120^{\circ}$, an inversely proportional relationship in LCL length was observed in both knee conditions. Compared to the intact knee, the LCL length decreased between $90^{\circ}$ and $120^{\circ}$ in the MCL-removed knee, which was associated with reversal of posterior translation of lateral cartilage contact in the anterior direction and medial translation of medial cartilage contact in the lateral direction.

MCL length decreased between $60^{\circ}$ and $90^{\circ}$ in the intact knee but increased in the knee with the LCL removed in an inversely proportional relationship. Increased MCL length also reversed medial translation of the medial cartilage contact to lateral translation (Fig. 5). 


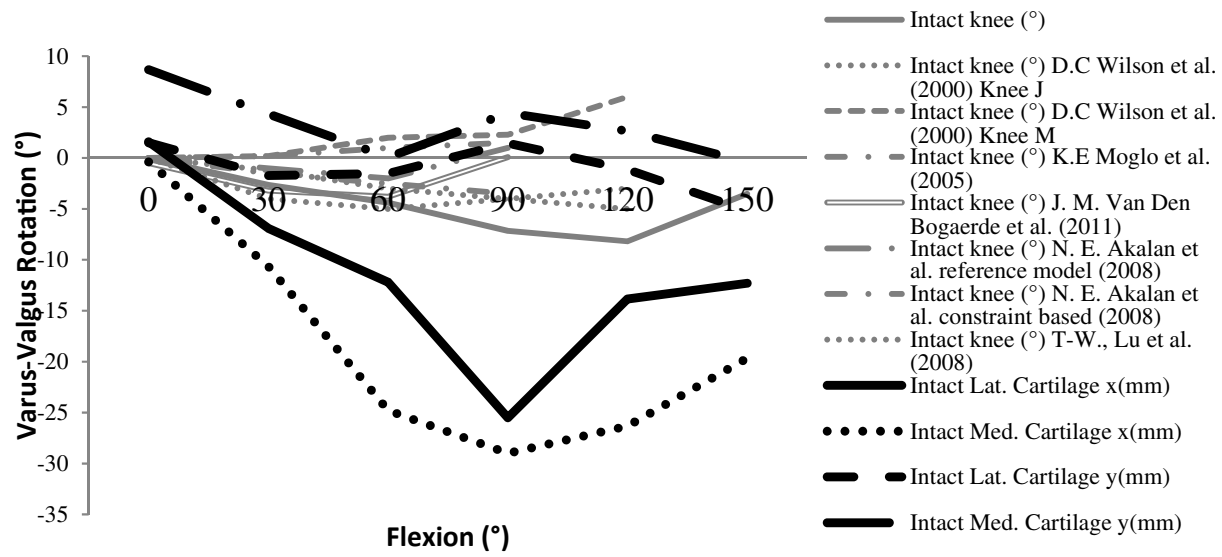

(a)

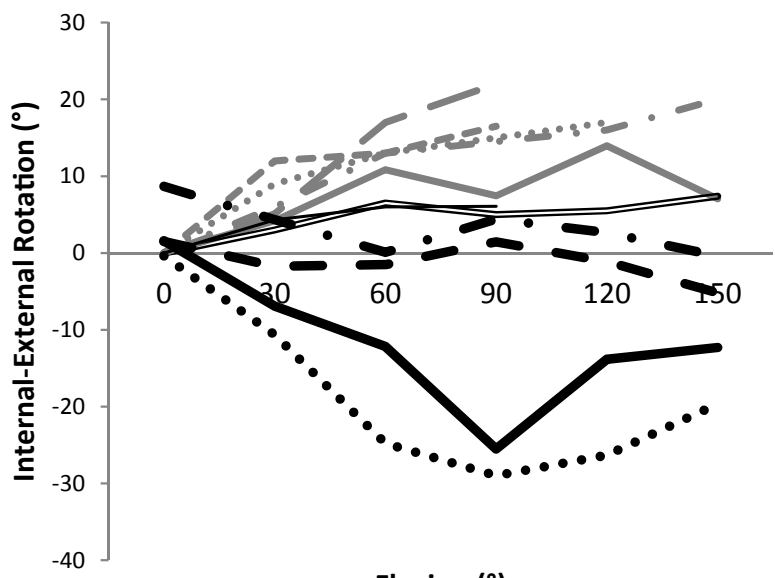

Flexion $\left({ }^{\circ}\right)$

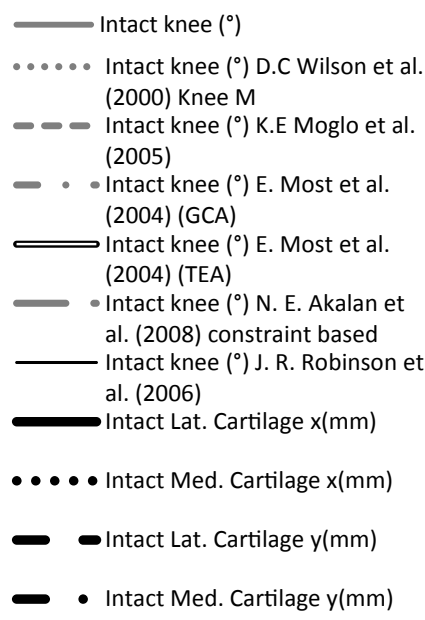

(b)

Fig. 7. Comparison of tibial rotations with previous studies. ${ }^{6,14,17-21}$ (a) VV rotation and (b) IE rotation.

Increases in ligament length may result in an increase in ligament tension. When the tension was increased, the ligament functioned as a dominant stabilizer of the joint. However, the observed inversely proportional relationship in ligament length changes between knees with intact and removed ligaments was surprising. A comparison of translational and rotational tibial movements and changes in ligament length suggest that the LCL or MCL may be loaded during translation and rotation of the intact knee (Figs. 3-5). When the MCL or LCL was removed, the observed motion was opposite to that seen in the intact knee. Therefore, the ligament that was loaded in the intact knee was not loaded when the opposite collateral ligament was removed. This also caused opposite translation of lateral and medial cartilage 
contact points, which is directly related to the transfer of compressive loading from ligaments to the contact surface area. When a collateral ligament is cut, the intersection of contact point pairs moves away from the ligament site.

The LCL length change trajectory observed between $0^{\circ}$ and $90^{\circ}$ was similar to that seen by Bergamini et al.,${ }^{22}$ Victor et al.,${ }^{23}$ Hsieh and Draganich, ${ }^{24}$ and Ghosh et al. ${ }^{25}$ and between $0^{\circ}$ and $120^{\circ}$ the LCL showed a similar length change trajectory with the middle portion of the LCL in an intact knee reported by Hosseini et al. ${ }^{26}$ The MCL length change trajectory was similar to the superficial MCL length trajectory published by Victor et al. ${ }^{23}$ and the superficial anterior and deep middle portions MCL length trajectory reported by Hosseini et al. ${ }^{26}$ (Fig. 8).

Most biomechanical studies on knee joints and surrounding tissue behavior are based on experiments or simplified joint models. ${ }^{27,28}$ However, it is difficult to make direct comparisons between the reported experimental or modeling-based studies, which differ in kinematic modeling methods, test structure, mechanical properties, and bone surface geometry. Experimental kinematic analysis of joints is usually based on anatomical landmarks that limit reproducibility. ${ }^{29}$ Moreover, simplified anatomical joint models are described that have reduced DOF and cannot provide six DOF kinematic information and ligament length changes.

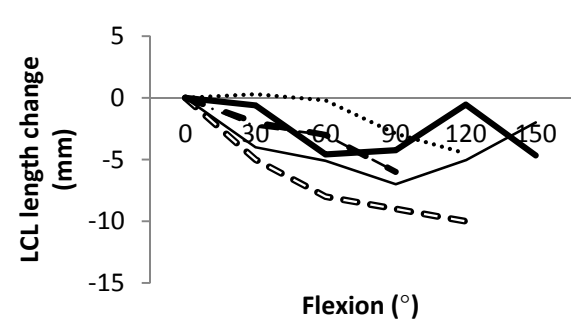

(a)

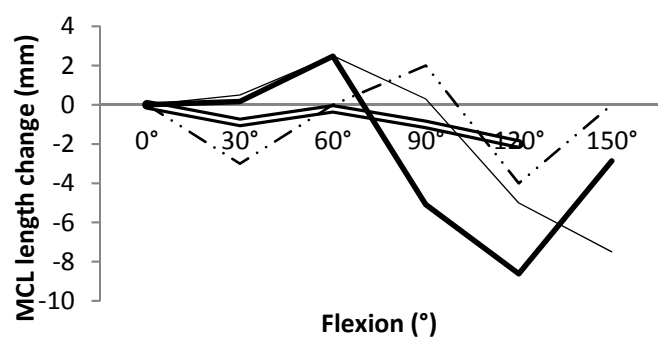

(b)
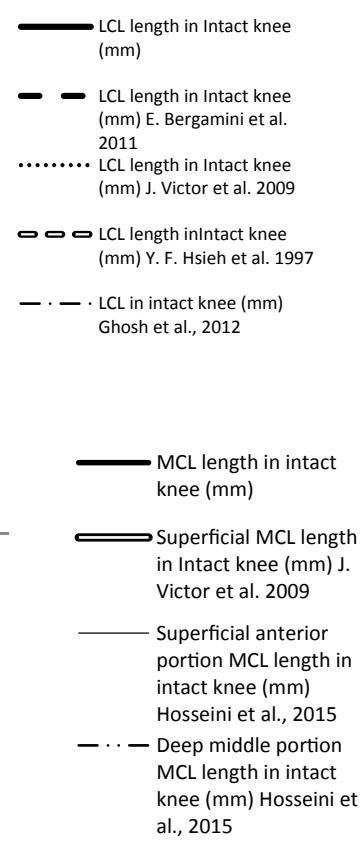

al., 2015

Fig. 8. Comparison of ligament length changes with previous studies. ${ }^{22-26}$ (a) LCL length change and (b) MCL length change. 
Although a number of published studies report the natural and altered kinematics of the tibia relative to the femur, this is the first report of six DOF knee joint kinematics, changes in collateral ligament lengths, and changes in cartilage contact positions in the context of MCL and LCL rupture.

\section{Conclusions}

Here we evaluated, for the first time, the effect of MCL and LCL rupture on six DOF knee joint kinematics, changes in ligament length, and changes in cartilage contact positions. These data have implications for knee arthroplasty research on collateral ligament injuries, cartilage contact problems, restoring ligament tension, and contact and wear problems with artificial joints. The total modeling and analysis time of a knee joint using the musculoskeletal joint modeler software was approximately two hours. Although musculoskeletal joint modeling based on multi-body dynamics may be faster than finite-element-based software, the efficacy still needs to be improved to make it practical and user-friendly for clinical use. A greater focus from clinicians and researchers on joint modeling and the use of modeling-based results in clinical practice may provide insights into predicting individual joint behavior after treatment, which may be difficult to observe and predict experimentally. Furthermore, kinematic analysis using surface geometry may be applied to different types of joint replacement implants.

\section{Acknowledgment}

This study was supported in part by Teknopark at Eastern Mediterranean University.

\section{References}

1. Farrokhi S, Voycheck CA, Klatt BA, Gustafson JA, Tashman S, Fitzgerald GK, Altered tibiofemoral joint contact mechanics and kinematics in patients with knee osteoarthritis and episodic complaints of joint instability, Clin Biomech 29:629-635, 2014.

2. Li G, DeFrate LE, Park SE, Gill TJ, Rubash HE, In vivo articular cartilage contact kinematics of the knee: An investigation using dual-orthogonal fluoroscopy and magnetic resonance image-based computer models, Am J Sports Med 33:102-107, 2005.

3. Kazemi M, Dabiri Y, Li LP, Recent advances in computational mechanics of the human knee joint, Comput Math Methods Med 2013:718423, 2013.

4. Kiapour A, Kiapour AM, Kaul V, Quatman CE, Wordeman SC, Hewett TE, Demetropoulos CK, Goel VK, Finite element model of the knee for investigation of injury mechanisms: Development and validation, J Biomech Eng 136:011002, 2014.

5. Muller JH, Simulating in-vivo tibiofemoral loads with the aid of a customised LifeModeler musculoskeletal system, ASME 2012 Summer Bioengineering Conf, 2012, pp. $195-196$.

6. Van den Bogaerde JM, Shin E, Neu CP, Marder RA, The superficial medial collateral ligament reconstruction of the knee: Effect of altering graft length on knee kinematics and stability, Knee Surg Sports Traumatol Arthrosc 19(Suppl 1):S60-68, 2011. 
7. Defrate LE, Sun H, Gill TJ, Rubash HE, Li G, In vivo tibiofemoral contact analysis using 3D MRI-based knee models, J Biomech 37:1499-1504, 2004.

8. Wang H, Chen T, Koff MF, Hutchinson ID, Gilbert S, Choi D, Warren RF, Rodeo SA, Maher SA, Image based weighted center of proximity versus directly measured knee contact location during simulated gait, J Biomech 47:2483-2489, 2014.

9. Ozada N, A novel musculoskeletal joint modelling for orthopaedic applications, Ph.D. Thesis, Brunel University Research Archive Web site, 2008, Available at http://bura. brunel.ac.uk/handle/2438/6556 [accessed on 10 June 2015].

10. Várady T, Facello MA, Terék Z, Automatic extraction of surface structures in digital shape reconstruction, CAD Comput Aided Des 39:379-388, 2007.

11. Mirtich B, Fast and accurate computation of polyhedral mass properties, J Graph Tools 1:31-50, 1996.

12. Westphal CJ, Schmitz A, Reeder SB, Thelen DG, Load-dependent variations in knee kinematics measured with dynamic MRI, J Biomech 46:2045-2052, 2013.

13. Mesfar W, Shirazi-Adl A, Biomechanics of the knee joint in flexion under various quadriceps forces, Knee 12:424-434, 2005.

14. Akalan NE, Özkan M, Temelli Y, Three-dimensional knee model: Constrained by isometric ligament bundles and experimentally obtained tibio-femoral contacts, J Biomech 41:890-896, 2008.

15. Kaiser J, Bradford R, Johnson K, Wieben O, Thelen DG, Measurement of tibiofemoral kinematics using highly accelerated 3D radial sampling, Magn Reson Med 69:1310-1316, 2013.

16. Li G, Moses JM, Papannagari R, Pathare NP, DeFrate LE, Gill TJ, Anterior cruciate ligament deficiency alters the in vivo motion of the tibiofemoral cartilage contact points in both the anteroposterior and mediolateral directions, J Bone Joint Surg Am 88:1826$1834,2006$.

17. Wilson DR, Feikes JD, Zavatsky AB, O'Connor JJ, The components of passive knee movement are coupled to flexion angle, J Biomech 33:465-473, 2000.

18. Moglo KE, Shirazi-Adl A, Cruciate coupling and screw-home mechanism in passive knee joint during extension-Flexion, J Biomech 38:1075-1083, 2005.

19. Most E, Axe J, Rubash H, Li G, Sensitivity of the knee joint kinematics calculation to selection of flexion axes, J Biomech 37:1743-1748, 2004.

20. Robinson JR, Bull AMJ, Thomas RRD, Amis AA, The role of the medial collateral ligament and posteromedial capsule in controlling knee laxity, Am J Sports Med 34:1815-1823, 2006.

21. Lu T-W, Tsai T-Y, Kuo M-Y, Hsu H-C, Chen H-L, In vivo three-dimensional kinematics of the normal knee during active extension under unloaded and loaded conditions using single-plane fluoroscopy, Med Eng Phys 30:1004-1012, 2008.

22. Bergamini E, Pillet H, Hausselle J, Thoreux P, Guerard S, Camomilla V, Cappozzo A, Skalli W, Tibio-femoral joint constraints for bone pose estimation during movement using multi-body optimization, Gait Posture 33:706-711, 2011.

23. Victor J, Wong P, Witvrouw E, Sloten J Vander, Bellemans J, How isometric are the medial patellofemoral, superficial medial collateral, and lateral collateral ligaments of the knee? Am J Sports Med 37:2028-2036, 2009.

24. Hsieh YF, Draganich LF, Increasing quadriceps loads affect the lengths of the ligaments and the kinematics of the knee, J Biomech Eng 120:750-756, 1998.

25. Ghosh KM, Merican AM, Iranpour F, Deehan DJ, Amis AA, Length-change patterns of the collateral ligaments after total knee arthroplasty, Knee Surg Sport Traumatol Arthrosc 20:1349-1356, 2012. 
26. Hosseini A, Qi W, Tsai T-Y, Liu Y, Rubash H, Li G, In vivo length change patterns of the medial and lateral collateral ligaments along the flexion path of the knee, Knee Surg Sports Traumatol Arthrosc 23:3055-3061, 2014.

27. Qi W, Hosseini A, Tsai TT-Y, Li J-SJJ-S, Rubash HEH, Li G, In vivo kinematics of the knee during weight bearing high flexion, J Biomech 46:1576-1582, 2013.

28. Feeley BT, Muller MS, Allen AA, Granchi CC, Pearle AD, Biomechanical comparison of medial collateral ligament reconstructions using computer-assisted navigation, $\mathrm{Am} \mathrm{J}$ Sports Med 37:1123-1130, 2009.

29. Morton NA, Maletsky LP, Pal S, Laz PJ, Effect of variability in anatomical landmark location on knee kinematic description, J Orthop Res 25:1221-1230, 2007. 